\title{
Entrelacs
}

Cinéma et audiovisuel

$14 \mid 2018$

Marchés du film : évolutions, mutations et perspectives

\section{Le marché de l'animation télévisée au Japon : Une omniprésence du licensing et quelques exceptions culturelles}

\section{Samuel Kaczorowski}

\section{(2) OpenEdition}

\section{Journals}

Édition électronique

URL : http://journals.openedition.org/entrelacs/4300

DOI : 10.4000 /entrelacs.4300

ISSN : 2261-5482

Éditeur

Éditions Téraèdre

Référence électronique

Samuel Kaczorowski, « Le marché de l'animation télévisée au Japon : Une omniprésence du licensing et quelques exceptions culturelles », Entrelacs [En ligne], 14 | 2018, mis en ligne le 16 décembre 2018, consulté le 10 décembre 2020. URL : http://journals.openedition.org/entrelacs/4300 ; DOI : https:// doi.org/10.4000/entrelacs.4300

Ce document a été généré automatiquement le 10 décembre 2020.

Tous droits réservés 


\title{
Le marché de l'animation télévisée au Japon : Une omniprésence du licensing et quelques exceptions culturelles
}

\author{
Samuel Kaczorowski
}

1 Si les ressemblances extérieures entre les films de cinéma et les séries télévisées sont de plus en plus fortes, les méthodes de productions des séries d'animation accusent encore des différences fondamentales qui ont trait à leurs modes de financement. Au Japon, cette dépendance a atteint, dans les années 1960, des proportions telles qu'elle caractérise désormais le fonctionnement de ce marché.

2 Alors qu'en Europe, les sociétés de production de séries d'animation attendent souvent des chaînes qu'elles s'engagent financièrement dans la réalisation des films, le Japon ne peut guère compter, depuis les premières séries lancées par Osamu Tezuka au milieu de l'ère Shōwa ${ }^{1}$, que sur un licensing ${ }^{2}$ privé qui infléchit l'ensemble de la production et, ce faisant, le paysage télévisuel. Le licensing renvoie ici à une cession de licence de marque par le détenteur des droits à un tiers moyennant en échange une redevance.

3 Outre que les chaînes japonaises privées ne coproduisent jamais les anime ${ }^{3}$ qu'elles diffusent, les studios de production doivent, en sus, « acheter » des plages d'antenne pour diffuser leurs films. Pour viabiliser ces marchés, les grands groupes privés tels que les fabricants de jouets ou de jeux vidéo (ex. Bandai, Tomy, Sony, Nintendo) trouvent un intérêt évident à devancer les studios, à familiariser puis à fidéliser le jeune public à quelques héros de fiction facilement déclinables en figurines et autres produits dérivés.

4 Au Japon, les logiques du manga ${ }^{4}$ et de l'animation partagent une histoire commune ${ }^{5}$ : non seulement les anime sont presque tous adaptés de mangas ${ }^{6}$, mais en plus, le montage financier du produit télévisuel fut calqué, en premier lieu, sur celui de l'industrie bédéistique. Pour comprendre les difficultés des marchés actuels à s'extraire de ces anciennes logiques, il semble intéressant de s'appuyer sur des données macroéconomiques ${ }^{7}$ pour ensuite s'attacher à l'étude du cas particulier de Mushi productions ${ }^{8}$ 
, un de ses berceaux, notamment à travers le témoignage des survivants ${ }^{9}$ de l'époque des premières négociations financières autour des séries télévisées.

\section{Un processus de production dépendant du Licensing} propres $^{10}$, dans le milieu de l'animation, peu d'entreprises en ont la capacité. Pour qu'une série soit diffusée sur une chaîne de télévision, il faut généralement que cette dernière en ait acheté au moins une saison aux studios qui en sont les producteurs ${ }^{11}$.

6 Au Japon les difficultés des producteurs d'animation sont similaires à celles rencontrées lors de la création d'un épisode pilote. La mainmise des fabricants de jouets reporte toute l'attente des studios sur les ventes des produits dérivés qui versent un tantième dérisoire au titre des droits d'auteur (qui d'ailleurs, revient le plus souvent à l'auteur du manga original que l'anime ne fait qu'adapter). Ce faisant, la proximité entre producteurs de films et fabricants de jouets s'accroit, au point parfois de se télescoper. Sur les quinze dernières années, le ratio entre les recettes générées par les produits d'exploitation et celles relatives à la seule diffusion des films sur différents supports montre une présence de plus en plus nette des professionnels du licensing ${ }^{12}$.

Figure 1 : Recettes générées pour l'ensemble des productions et des produits de l'animation télévisée japonaise pour l'année 2016 (milliards de yens)
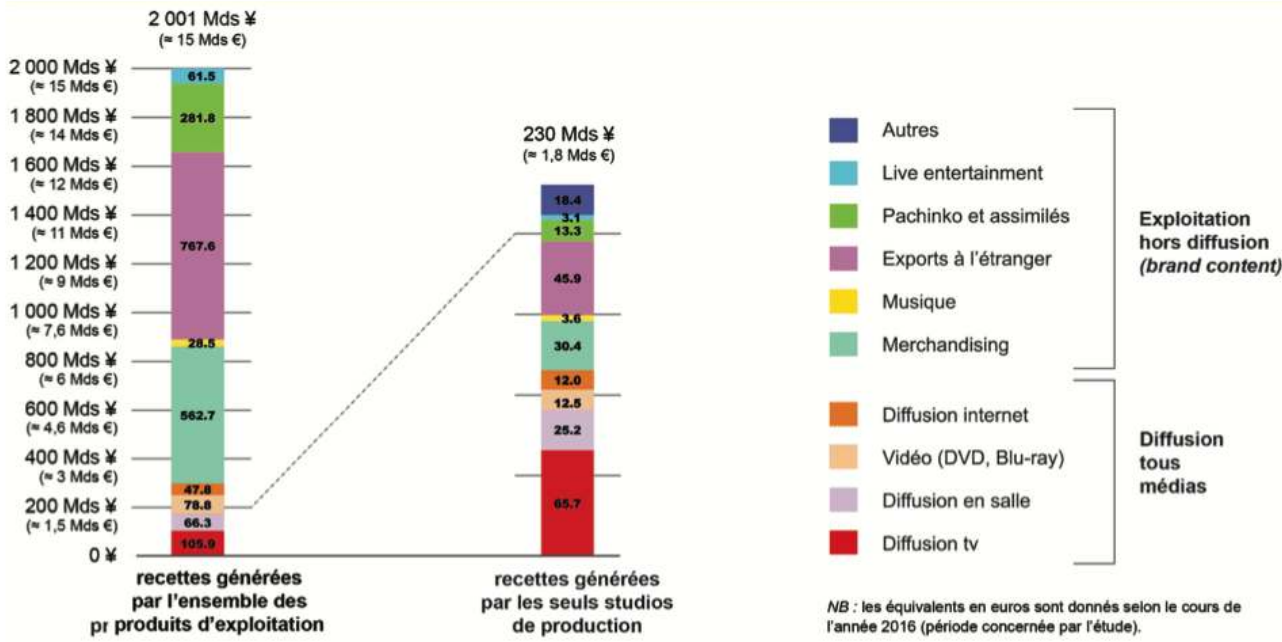

(c) Samuel Kaczorowski (source Hirochimi Masuda).

Dans certains cas, le fabricant de jouets initie la série. Son équipe marketing imagine sommairement une fiction de nature à mettre en avant des produits consommables (cartes à jouer, badges, figurines, déguisements...), puis contacte des professionnels des médias (auteurs de mangas ou d'anime) ou démarche les studios d'animation à Nerima, la «Mecque » du dessin animé, dans l'espoir d'une alliance et d'une signature de contrat pour, au minimum, une première saison. Dans d'autres cas, les fabricants de jouets développent leur propre studio de production audiovisuelle (généralement fusionné avec l'équipe de développement des logiciels et jeux vidéo) ${ }^{13}$.

8 Au regard des bénéfices que perçoivent les producteurs d'anime sur la vente des jouets, les DVD constituaient, jusqu'il y a peu, la rente la plus intéressante d'un studio de 
production, parce que la moins amputée à la source. Mais avec le développement de l'internet et de la technologie de diffusion en streaming, la vente de DVD ou de Blu-ray a considérablement chuté ${ }^{14}$. Le phénomène eut pour effet de renforcer encore les liens entre les studios de productions et les multinationales mandataires : on ne s'étonnera guère du nombre accru des films à forte incitation commerciale. Le phénomène est si caricatural que certains producteurs ne prennent plus la peine de dissimuler le produit derrière une histoire prétexte: c'est désormais lui qui occupe le premier rang de l'intrigue. Pour exemple, la série à succès $\mathrm{Yu}$-Gi-Oh ${ }^{15}$ ne montre guère que des adolescents qui combattent au moyen de cartes à jouer, celles-là mêmes que les jeunes peuvent trouver dans le commerce, à des enseignes aussi variées que nombreuses.

Certains groupes financent des programmes à vocation culturelle en y associant leur image (Sony est passé de messages publicitaires sur le matériel électronique à des programmes sur la communication entre les hommes; IBM Japon promeut les programmes sur l'art...), mais la frontière entre spots intéressés et mécénat se réduit.

Les émissions, les programmes et en conséquence la chaîne de télévision tout entière bénéficieront du prestige que leur confère [un] partenariat [avec des sponsors]. Cette dynamique est à l'origine du développement d'un marché publicitaire japonais qui encourage l'existence de programmes culturels sur les chaînes privées [...] Mais en contrepartie [...], les émissions et les programmes portent de plus en plus la marque de ceux qui les ont financés ${ }^{16}$.

De fait, lorsque j'ai rencontré Thomas Romain ${ }^{17}$, auteur de la série d'animation Basquash aux studios Satelight de Nerima, celui-ci dessinait des cartes de collection à l'effigie des héros de la série pour accompagner le DVD et relancer sa vente, après la diffusion télévisée. Si de plus en plus de fabricants de jouets préfèrent élargir leurs activités jusqu'à la conception et la réalisation d'anime, de plus en plus de studios d'animations ont, à l'inverse, tendance à fabriquer les produits dérivés par eux-mêmes. Les cartes de collection ou les décors de pachinko ${ }^{18}$ sont commodes : leur production n'exige aucune technologie hétérodoxe à la logique du dessin animé. Ce sont les objets les plus directement accessibles et faciles à lancer sur le marché. Les images fabriquées pour décorer les pachinko produisent du bénéfice en elles-mêmes, mais c'est aussi une publicité indirecte pour les séries dont elles reprennent l'univers. Presque tous les studios s'y adonnent, et certains départements des studios Satelight sont entièrement dévolus à la gestion et à la fabrication de ces produits, ainsi qu'à d'autres dérivés des films. Dans certains étages du bâtiment qui m'a accueilli pendant plusieurs semaines pour suivre la production de la série Fairy tail ${ }^{19}$, les activités sont tenues secrètes. Officiellement, on y effectue les négociations relatives au licensing et on parle des futurs projets, mais le lieu est soustrait à la fréquentation d'une grande majorité des employés: l'exclusivité commerciale fait la loi.

11 Dans la période faste du début des années 1990, l'anime représentait environ 6\% de l'ensemble des programmes des six principales chaînes hertziennes de Tokyo (soit une trentaine d'heures hebdomadaires). Depuis, il ne cesse de perdre du terrain sous l'effet des pressions marketing. Le traitement de l'animation par les chaînes de télévision, si peu favorable sur le plan financier, s'est dessiné progressivement dans les années 1960 face à une offre croissante, au départ de laquelle on trouve le mangaka à succès et cinéaste d'animation Osamu Tezuka. Retour sur les premières négociations financières : celle qui scellèrent un destin financier de plusieurs décennies, décidément bien chiche. 


\section{Le cas de Mushi Productions}

12 À l'époque des toutes premières séries d'animation de l'histoire, le forfait tarifaire pour la série télévisée négocié par Mushi productions (ou Mushi pro), le studio fondé par Osamu Tezuka en 1961, ne pouvait être établi sur des bases existantes puisque la situation était nouvelle. La comparaison avec les coûts des séries dramatiques (ou drama, au Japon) traditionnelles avait tellement peu à voir avec l'animation qu'on ne pouvait pas reporter le ratio entre temps de production et temps d'antenne pour établir un devis. Dans les «standards» de l'industrie du film cinématographique (aux États-Unis ou encore aux studios Toei de Tokyo qui, au niveau de l'organisation, en était le décalque), 30 minutes d'animation nécessitaient l'action d'environ 100 personnes pendant 6 mois, ce qui s'estimait à 30000000 yens. C'est soixante fois plus que le coût de réalisation d'une émission de télévision pour enfant filmée en live, qui coûtait en moyenne 500000 yens. Entre ces deux, Osamu Tezuka souhaitait se situer à mi-chemin.

La chaîne publique NHK $^{20}$ (Nippon Hoso Kyokai) contacta Osamu Tezuka pour lui commander l'écriture de scénarios pour un feuilleton filmé en live à partir de l'intrigue principale de son manga à succès Le monde utopique de Lurue. Le programme Fushigina shonen (le garçon mystérieux) fut tourné en studio puis diffusé à partir de mai 1961. C'est un format semblable à celui-ci que Tezuka allait reprendre pour l'animation. Les tarifs ordinaires de 500000 yens $^{21}$ furent à peine augmentés du fait de la technique utilisée et de l'indépendance de Mushi pro par rapport aux chaînes. Un épisode de 30 minutes se monnaya ainsi 550000 yens $^{22}$, générique compris. Dès les premières diffusions, les fabricants de jouets et de textile sont venus négocier des licences pour vendre des produits à l'image du héros du dessin animé, déjà rendu populaire par un manga décennal.

La législation japonaise en matière de propriété intellectuelle et de protection des œuvres via le droit de l'image présentait un tel flou juridique que l'industrie du manga, avant celle de l'anime perdait déjà une part importante de sa rentabilité. En effet, la notion de droit d'auteur n'existe juridiquement au Japon que depuis le début des années 1990 et demeure imprécise, aujourd'hui encore, sur les montants éligibles et la part reversée à l'artiste. L'usage consiste à laisser chaque société fixer sa propre "politique » par voie contractuelle. Les créations pirates qui singeaient le style de Tezuka étaient nombreuses et constituaient un manque à gagner non négligeable pour Mushi pro, qui produisait conjointement films et bandes dessinées ${ }^{23}$. Finalement, en animation, la loyauté des partenaires industriels soutenait les œuvres certainement plus efficacement que la loi et évitait, du moins au début, les dérives observées dans le manga ${ }^{24}$. Parmi ces partenaires, on retiendra les chocolats à l'effigie de l'enfant-robot Atom (série d'animation Tetsuwan Atom), vendus avec une série d'autocollants par la firme agro-alimentaire Meiji Seika, ainsi que les spots publicitaires de la firme Sanyo electric qui utilisaient la mélodie du générique de Tetsuwan Atom.

15 Ce montage néanmoins fragile n'empêcha pas la faillite de Mushi pro, qui survint en 1973, cinq ans après le départ d'un Tezuka si endetté que les créanciers menaçaient de le déposséder de ses droits sur ses propres œuvres. Tezuka reçut alors un coup de pouce, comme tombé du ciel. Kenzo Kasai, gérant de la société Aprica qui produisait du mobilier pour enfant, vint le trouver à Tokyo. Vingt ans auparavant, quand Tetsuwan Atom n'avait pas encore conquis la télévision, Kasai écrivit à Tezuka pour demander l'autorisation 
d'utiliser l'image du personnage d'Atom sur le mobilier tubulaire de l'usine de son père, alors en très grande difficulté financière. Grâce à l'accord de Tezuka, l'entreprise repartit, florissante. Quand en 1970, Kasai apprit la détresse de Tezuka, il lui proposa de racheter la totalité de ses droits pour empêcher la mainmise des créanciers. Dix ans plus tard, Kasai restituait la totalité des droits à l'auteur. Tezuka conserva ainsi la propriété légale de ses productions, mais le studio Mushi pro fut tout de même racheté en 1977 par MP corporation.

16 Ainsi, le principe de financement du dessin animé par les produits dérivés représente une forme de modèle pour les studios, y compris ceux qui ne peuvent s'assoir sur le succès passé des mangas ou sur la gloire médiatique de leur fondateur.

\section{Une exception culturelle}

17 La législation et la réalité économique de l'anime au Japon repose désormais essentiellement sur les ventes de supports multimédias et de produits dérivés. C'est sans doute pour contrer le nivellement mercantile du sponsoring que la chaîne de télévision NHK, reconnue comme très importante dans le milieu de l'animation japonaise, fait exception en promouvant cet art sous sa forme mineure et expérimentale. Elle échappe à la règle générale énoncée plus haut en aidant bel et bien au financement des anime. Cela se produit sous deux formes :

18 - Soit le studio producteur de la série est la propriété du groupe NHK. Les artistes qui y travaillent sont salariés. Dans ce cas, les séries créditent NHK au titre des producteurs à la fois exécutifs et délégués. Elles sont, en quelque sorte, «labellisées», c'est-à-dire diffusées sous une licence éducative et artistique ;

19 - Soit la série est le résultat d'une expérience artistique menée en partenariat avec des auteurs, plasticiens et techniciens en résidence, ou bien des écoles. Cela donne, généralement, des séries courtes, des courts métrages, ou bien des publicités.

Le financement de la programmation publique reste bien intégré à la marche générale de la NHK et à ses préoccupations structurelles. Il peut ainsi concerner toutes les étapes, de la production à la programmation, dans la mesure où, par exemple, la NHK fait rarement appel à des sociétés extérieures pour sous-traiter ses travaux de réalisation ${ }^{25}$.

21 Même si la structure administrative de NHK reste normalement hiérarchisée, les responsables jouissent d'un pouvoir décisionnel certainement plus large et moins fragmenté que sur les chaînes privées. De fait, les productions animées produites par NHK vont de l'animation la plus traditionnelle en termes de technique et de signature (Taiyo no ko Esteban - Les mystérieuses cités d'or - est un feuilleton franco-japonais, d'abord diffusé sur NHK puis sur Antenne 2 à partir de septembre 1983) à l'animation en papier découpé, pâte à modeler, craie, sable rétro-éclairé... à destination des enfants, adolescents, et même des adultes. Une diversité qui démentirait les préjugés européens sur les fameux "standards japonais » si toutefois plus d'un pour cent de ces films parvenait jusqu'en Europe. Le fait est que la relative désinhibition du Japon actuel quant à aux images permises à la télévision aurait de quoi surprendre le public occidental. L'offre de la télévision japonaise va des spots publicitaires artistiquement ébouriffants aux feuilletons scatologiques de trente minutes et à la facture quasi amateur. Mais encore une fois, même si la bienveillance culturelle du groupe NHK est historique, elle n'aurait pu proposer une formule avantageuse à Tezuka qu'à la double condition de croire à son 
projet de feuilleton d'animation et de vérifier l'existence d'antécédents, ce qui, en tout état de cause, était impossible.

\section{L'animation « indépendante »}

Aujourd'hui, les séries de marionnettes filmées en stop-motion (image par image), selon un procédé et un temps de production proches de ceux du dessin animé peuvent également s'inviter sur le petit écran, tandis qu'elles étaient surtout employées, dans les années 1960, pour les publicités. C'est notamment ce secteur qu'investit, depuis longtemps et loin des préoccupations télévisuelles, l'une des plus célèbres personnalités de l'animation indépendante au Japon : Koji Yamamura.

$\mathrm{Au}$ début de ma carrière, j'ai travaillé quelques fois pour des commandes de télévision [...] j'estime avoir pu réaliser des films proches de ce qu'est mon style personnel dans l'absolu. Cela dit, je suis conscient que j'ai sans doute une chance particulière ${ }^{26}$.

La longévité télévisuelle de Yamamura est très certainement due à sa notoriété. Parmi ses films de télévision, notons la trilogie des Karo to Piyobuputo ${ }^{27}$ (Karo et Piyobupt), et Babel no $\mathrm{Hon}^{28}$ (le livre de Babel). À l'époque de leurs premières diffusions, les productions de Yamamura étaient déjà assimilées par un certain public, au cinéma d'auteur, «une perspective qui me convenait et que j'assumais. ${ }^{29}$ "

C'est peut-être le succès d'audience de ces films, mais plus probablement leur originalité graphique qui retint l'attention des directeurs des programmes de NHK pour la carte blanche de 2009, qui permit à l'artiste de travailler dans les conditions d'une résidence d'artiste pour une série de productions audiovisuelles à propos desquelles on ne lui demanderait rien, pourvu qu'il respecte les règles de bienséance inhérantes aux programmes «tout publics ». Diffusés à la galerie Uplink Tabera cafe dans district de Shibuya (Tokyo) en novembre 2009, les films en papier découpé, dessins animés sur cellulos, animation de marionnettes et de pâte à modelé disposée sur plaques de verre devant des décors peints... présentaient un format capable d'exploser, à lui seul, les grilles de programmes classiques. La durée de certains de ces objets les assimilait à un spot publicitaire (mais qui n'aurait, pour le coup, rien à vendre), tandis que leur déclinaison les rapprochait davantage de la mini-série. Selon leur tonalité, on décida de diffuser ces films comme des jingles au milieu de programmes pour enfants; les séquences plus longues furent insérées dans l'émission jeunesse Okasan to issho (" Avec maman »). 
Image 1 : Koji Yamamura, Babel no hon (le livre de Babel -photogramme issu du film), 1996

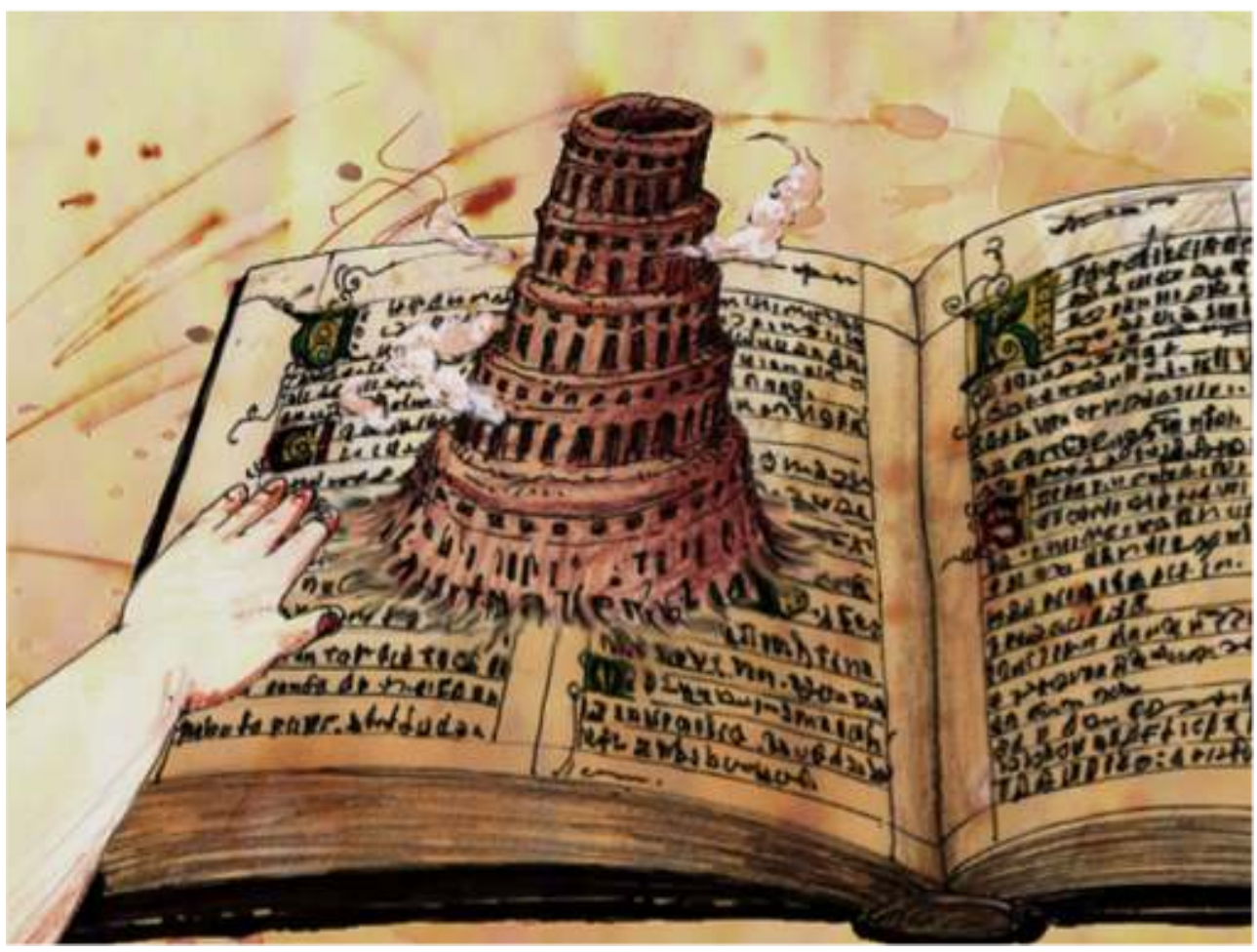

(c) Yamamura animation

Image 2 : Koji Yamamura, Karo to Piyobuputo - sandwiches (Karo et Piyobupt - sandwiches photogramme issu du film), 1993.

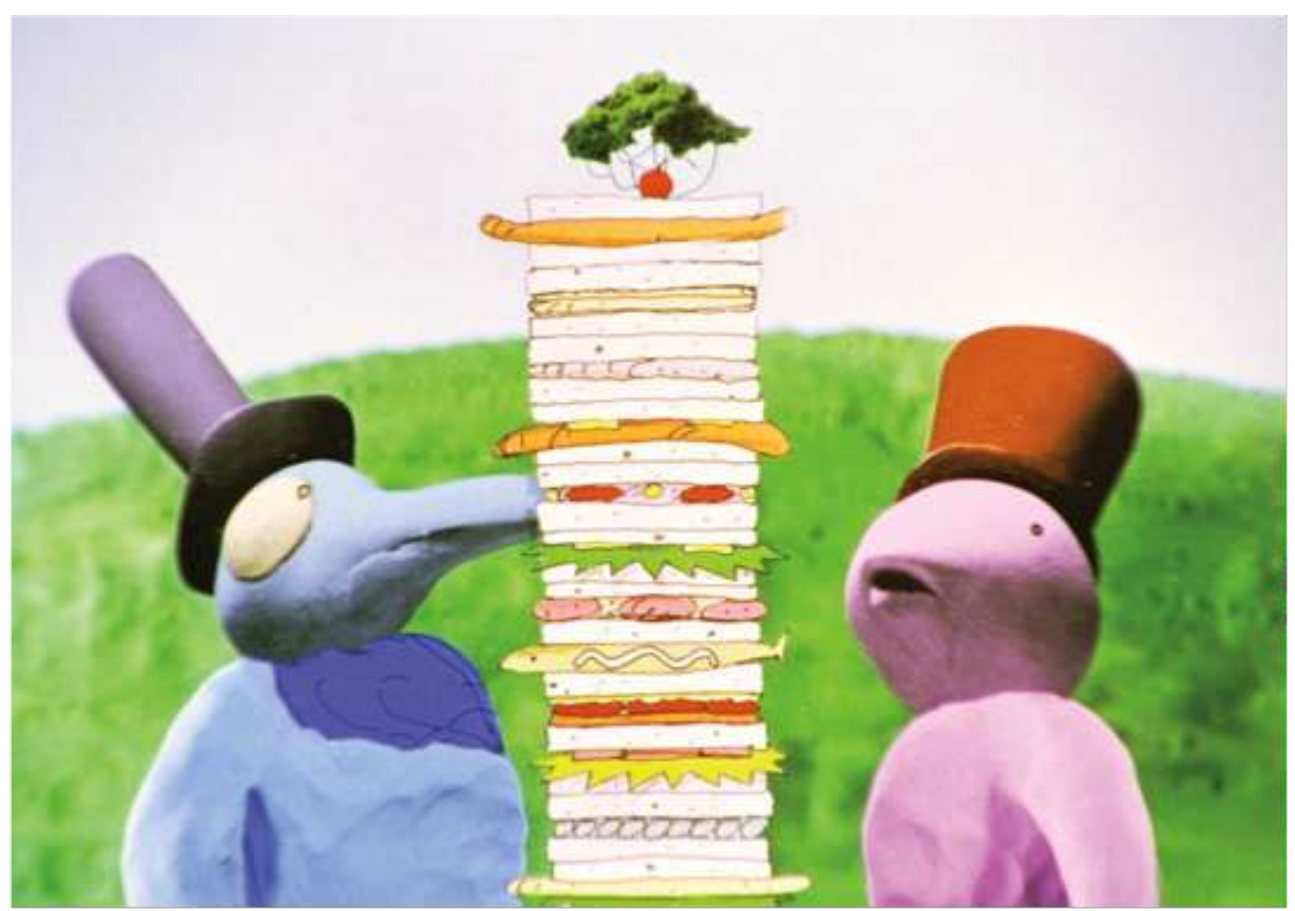

(c) Yamamura animation 
Mais cette «exception culturelle japonaise » qu'offre NHK mérite cependant d'être nuancée par sa relative marginalité et par son principe même: on n'offre une carte blanche qu'aux auteurs-artistes déjà reconnus pour leur œuvre, minimisant ainsi le risque financier. Même la diffusion des films expérimentaux des écoles d'animation japonaises ou les résultats des workshops publics ne peuvent se passer de la notoriété du ou des artistes désignés pour diriger les opérations de production (et la chaîne de bien prendre soin de fonder sa publicité sur ces noms). artiste de talent de ne se consacrer qu'à la fabrication de films reste, en 2018, très incertaine.

Ce qui me paraît important dans mon travail, c'est de permettre au public d'accéder aux films courts, de créer ces points de rencontres, ces « rendez-vous ». Pour moi, ce sont les films qui comptent avant tout. Je souhaite qu'on puisse les voir, les retenir, avant de s'intéresser à leur auteur. Si on veut focaliser sur le réalisateur par la suite, c'est très bien, mais c'est dans cet ordre-là que les choses doivent se passer. Or, quand on commence à être un peu connu, y compris au sein de ce public très restreint, c'est le processus inverse qui se met en place : les gens vont voir vos films précisément parce que ce sont les vôtres; ils délaissent la découverte pure, l'appréciation des qualités propres des films, leurs forces. C'est un phénomène qui, à mon sens, peut poser problème ${ }^{30}$.

\section{Conclusion}

La série d'animation prise en tenaille par les financeurs entre la notoriété des auteurs et l'obligation de multiplier les occasions de vente n'est pas un phénomène si nouveau que cela et n'affecte d'ailleurs pas que les produits de télévision. Pour réunir les fonds permettant de réaliser le long métrage d'animation Kaze no tani no Nausicaa (Nausicaa de la vallée du vent), Hayao Miyazaki avait dû décliner son scénario en bande dessinée pour prouver aux producteurs que le récit pouvait trouver un public.

Après l'intervention d'Osamu Tezuka sur le marché de l'animation, il est devenu difficile de proposer autre chose que des séries animées. Les producteurs se sont progressivement désintéressés de toutes les formes d'animations non destinées au format télévisuel. Dans mon cas personnel, je n'arrivais pas à faire produire mes films par exemple ${ }^{31}$.

Même aujourd'hui, installé aux commandes d'une structure qu'on croit florissante, Hayao Miyazaki avoue que les studios Ghibli ne tiendraient pas sans la recette des ventes des peluches de Totoro. Il esquisse cependant une solution, que d'aucun jugera amère et certainement acceptable qu'à la condition de savoir qu'on va bientôt "raccrocher » (à moins, bien sûr, de se sentir la force de refondre en profondeur les conditions de financement des séries - au sens où on l'entendait hier, mais en tenant compte des supports et des habitudes de consommation d'aujourd'hui).

Je ne travaille plus qu'à la réalisation de courts métrages ${ }^{32}$ diffusés uniquement dans la petite salle de projection du musée que j'ai fait construire à Mitaka ${ }^{33}$. On pourrait penser que c'est un business, mais ce que je cherche en réalité, c'est une rencontre directe avec le jeune public. D'ailleurs, je congédie tous les producteurs qui frappent à ma porte pour me demander d'éditer ces films en DVD. Je veux que mes films demeurent un rendez-vous unique, puis qu'ils se transforment progressivement en souvenir. Au fond, je crois que l'animation est et doit rester une sorte d'« héritage $»^{34}$. 


\section{Sitographie}

William Audureau, « Ubisoft et Electronic Arts, grands gagnants du marché du jeu vidéo » dans journal Le Monde pixels, [en ligne], mai 2015 [référence du 01/05/2018], url : www.lemonde.fr/ pixels.

Philippe Bonfils, Commission Supérieure Technique de l'Image et du Son, Dossiers techniques techniques d'animation [en ligne, référence du 18/06/2017], url : www.cst.fr.

CNC (Centre national du cinéma et de l'image animée), Code du cinéma et de l'image animée [en ligne, référence du 27 juillet 2018], url : https://www.cnc.fr/professionnels/reglementation/ tous-les-textes.

Guillaume Marty, Animemorial - Encyclopédie des animés [en ligne, consulté de septembre 2008 à avril 2017], url : http://www.animemorial.net.

Masuda Hiromichi (dir.), Anime Industry Report 2017, The Association of Japanese Animation [en ligne], mise à jour juillet 2017 [référence du 28/07/ 2018], url : http://aja.gr.jp.

Jacques Roméro, Mushi, Toei, la guerre du feu [en ligne, référence du 13/06/2017], url : http:// lib.yamato.free.fr/doc/MushiToeiLaGuerreDuFeu.pdf.'

\section{Entretiens et correspondance}

Entretien avec Koji Yamamura, le 07/11/2009 à la galerie Uplink Tabela caffe (district de Shibuya, Tokyo). Questions : Samuel Kaczorowski, traduction : Ilan Nguyen.

Retranscris en intégralité dans Samuel Kaczorowski, «L'CEuvre filmique d'Osmu Tezuka et son incidence sur le cinéma d'animation », Ph. B. dissertation, Université de Toulouse-le-Mirail, 2014, pp. 413-445.

Correspondance écrite avec Koji Yamamura le 22/07/2015 et les 24 et 26/01/2018 (non publiée).

Visite du studio Mushi productions et entretien avec Satoshi Ito (auteur, réalisateur, actuel directeur de Mushi productions et ancien collaborateur d'Osamu Tezuka), Chinami Namba (auteur, réalisateur, ancien employé de Mushi productions et collaborateur d'Osamu Tezuka), le 19/08/2010 à Fujimidai (district de Nerima, Tokyo). Questions : Samuel Kaczorowski, traduction : Christophe Caillet. Retranscris en intégralité dans Samuel Kaczorowski, Capter le moment fuyant, Paris, L'Harmattan, 2017, pp. 227-236.

Entretien avec Gisaburō Sugī, (auteur, réalisateur, ancien employé de Mushi productions et collaborateur d'Osamu Tezuka), le 19/08/2011 à 10h à Nishi Magome (district de Ōta, Tokyo). Questions : Samuel Kaczorowski, Christophe Caillet, traduction : Christophe Caillet.

Retranscris en intégralité dans Samuel Kaczorowski, Capter le moment fuyant, Paris, L'Harmattan, 2017, pp. 237-249.

\section{BIBLIOGRAPHIE}

Andrea Baricordi, Sabrina Tunesi et al., Anime: A Guide to Japanese Animation (1958 - 1988), Londres, Protoculture, 2001. 
Philippe Berthet et Jean-Claude Redonnet, l'Audiovisuel au Japon, Paris, PUF, 1992, pp. 65-66.

Thomas Lamarre, The Anime Machine: A Media Theory of Animation, Minneapolis, University of Minnesota Press, 2009.

Eli L. Levitan, Animation art in the commercial film, New York, Reinhold Pub Corp. 1960.

Pierre Albert et André-Jean Tudesq, Histoire de la radio-télévision, Paris, P.U.F. 1996.

Benjamin Simmenauer, «La série télévisée, un ars dominandi », dans Mode de recherche ${ }^{\circ} 19$ : Soft Power, Paris, I.F.M., 2013, pp. 38-48.

Susan Rubin, Animation, the art and the industry - Art \& design series, Englewood Cliffs, N.J. Prentice-Hall, 1984.

Osamu Tezuka, Boku wa mangaka (Je suis mangaka), Tokyo, Kadokawa bunko, 2000 (première parution en 1979).

Osamu Tezuka, Boku no manga jinsei (Ma vie de manga), Tokyo, Imawani Stoten, 1997.

Samuel Kaczorowski, Capter le moment fuyant - Osamu Tezuka et l'invention de l'animation télévisée, Paris, L'Harmattan, 2017.

Alan Weber, « l'Influence de la télévision sur le cinéma », dans Cinémaction n44, Paris, Cerf, 1987.

\section{NOTES}

1. Début des années 1960.

2. Ensemble des actions qui permettent de légiférer (autoriser, définir les conditions...) sur les droits de reproductions ou d'exploitations des œuvres protégées au titre du droit d'auteur. L'expression peut également s'étendre à la gestion des bénéfices ainsi générés. 3. Expression utilisée au Japon pour désigner les dessins animés dans leur ensemble, quelles que soient leurs origines. En Europe et en France, le terme fut importé pour caractériser les productions typiquement japonaises.

4. Signifiant littéralement « image dérisoire », le manga (parfois utilisé au féminin) désigne une bande dessinée japonaise. L'expression « mangaka », qui désigne un auteur de manga, tend à s'inviter de plus en plus naturellement dans la langue française.

5. Le succès commercial des fictions populaires de bandes dessinées, d'abord publiées sous la forme de périodique puis rassemblée en un album unique, offre quelques garanties aux producteurs quant au succès du feuilleton télévisé qui en fera l'adaptation.

6. La totalité des vingt séries d'animation les plus regardées entre 1963 et 2017 sont toutes adaptées de mangas.

7. Un rapport décennal sur le marché du film d'animation au Japon fut produit en 2017 par l'Association of Japanese Animations. Voir Hiromichi Masuda (dir.), Anime Industry Report 2017, The Association of Japanese Animation [en ligne], mise à jour juillet 2017 [référence du 28/07/ 2018], url : http://aja.gr.jp.

8. Visite du studio Mushi productions et entretien avec Satoshi Ito (auteur, réalisateur, actuel directeur de Mushi productions et ancien collaborateur d'Osamu Tezuka), Chinami Namba (auteur, réalisateur, ancien employé de Mushi productions et collaborateur d'Osamu Tezuka), le 19/08/2010 à Fujimidai (district de Nerima, Tokyo).

9. Parmi lesquels Yasuo Otsuka, Ito Satoshi, Sadao Tsukioka et Gisaburō Sugī (entretiens menés au Japon entre 2009 et 2010 dans le cadre de l'étude « L'CEuvre filmique d'Osmu 
Tezuka et son incidence sur le cinéma d'animation » (Ph. B. dissertation, Université de Toulouse-le-Mirail, 2014).

10. CNC (Centre national du cinéma et de l'image animée), Code du cinéma et de l'image animée [en ligne, référence du 27 juillet 2018], url : https://www.cnc.fr/professionnels/ reglementation/tous-les-textes (voir Article 211-74 modifié par délibération n²017/ CA/31 du 23 novembre 2017, article 10).

11. Constat effectué par le réalisateur Frédéric Clémençon dans les studios de production de films d'animation télévisés Marlou films (Bordeaux) en 2002.

12. En 2016, les recettes générées par les seuls studios ne représentent que $11,50 \%$ du produit total de l'exploitation incluant produits dérivés, supports musicaux, événementiels... (voir fig. 1). En 2002, les recettes générées par la diffusion des films représentaient un quart des revenus globaux. En 2016, les diffusions ne rapportent plus que 14\% des recettes (source Anime Industry Report 2017, op. cit.)

13. Le studio franco-japonais Ankama, par exemple, prend en charge aussi bien les jeux vidéo, l'édition de $\mathrm{BD} / \mathrm{manga} /$ comics, mais c'est aussi un studio d'animation. Depuis sa création en 2001, Ankama a progressivement regroupé ses activités autour de la France. À la date de notre visite des studios de Tokyo en 2010, le groupe produisait la série Wakfu pour la télévision française, avec un savoir-faire et une main d'œuvre majoritairement japonais.

14. Les ventes de supports vidéo génèrent une recette de 129,4 milliards de yen (963 million d'euros) en 2002 pour seulement 78,8 Mds $¥$ (606 million d'euros) en 2016, alors que le nombre de séries produites a, lui, pratiquement doublé (source Anime Industry Report 2017, op. cit.)

15. Littéralement « Roi du jeu » - série télévisée adaptée en 1998 du manga éponyme de Kazuki Takahashi.

16. Philippe Berthet et Jean-Claude Redonnet, l'Audiovisuel au Japon, Paris, PUF 1992, pp. 65-66.

17. En mars 2010. Thomas Romain est employé du studio d'animation et de jeux vidéo Satelight. Il est animateur, auteur et directeur artistique sur des séries comme Basquash, Code Lyoko ou Oban Star-Racers.

18. Le pachinko est une machine proche du flipper qui permet de jouer de l'argent en achetant des billes d'acier et en les insérant dans la machine tout en essayant d'infléchir leur trajectoire. L'addiction du public japonais (surtout masculin) à ces machines est difficilement concevable en France : le chiffre d'affaires de cette industrie se situe au troisième rang de l'économie des loisirs japonais, derrière les restaurants et le tourisme. Les cartons produits par les auteurs de manga ou d'anime destinés à décorer l'arrière-plan de ces machines constitue une rente non négligeable.

19. Série d'animation produite à partir de 2009 par les studios Satelight, à partir du manga éponyme de Hiro Mashima.

20. La NHK a commencé à émettre en 1925 en se basant sur la compagnie de radio BBC, et créé sa propre chaîne de télévision en 1953. Aujourd'hui encore, il s'agit de l'unique groupe de radio-télévision japonais de service public.

21. Ce qui équivaudrait à environ 3600 euros en 2018, en tenant compte de l'érosion monétaire et de l'inflation.

22. Environ 4000 euros en 2018.

23. Lorsque Osamu Tezuka démarra la réalisation de films d'animation, son studio put compter sur les bénéfices réalisés auparavant par les ventes des mangas. Ces derniers maintinrent l'entreprise à flot jusqu'à 1973. 
24. Dans les années 1950, les mangas à succès étaient impunément plagiés sans qu'aucune gratification ne soit adressée à l'œuvre originale (pas même une mention).

25. Philippe Berthet et Jean-Claude Redonnet, op. cit., p. 61

26. Extrait de l'entretien avec Koji Yamamura le 07/11/2009 à la galerie Uplink Tabela caffe (district de Shibuya, Tokyo) retranscris en intégralité dans Samuel Kaczorowski, « L'CEuvre filmique d'Osmu Tezuka et son incidence sur le cinéma d'animation », Ph. B. dissertation, Université de Toulouse-le-Mirail, 2014, pp. 413-445.

27. 1993. Polyptyque qui regroupe trois films courts de 4'20" tournés en $35 \mathrm{~mm}$ : Ochi (la maison), Sandwiches, et Ame no Hi (jour de pluie).

28. 1996. Film de 5'00" tourné en $16 \mathrm{~mm}$.

29. Koji Yamamura, ibidem.

30. Ibidem.

31. Entretien informel avec Hayao Miyazaki 28/08/2010 à 11h. Transcrit dans « L'œuvre filmique d'Osamu Tezuka et son incidence sur le cinéma d'animation », op. cit. p. 435.

32. Déclaration datant de 2010, quand Miyazaki pensait arrêter définitivement la réalisation de longs métrages.

33. Ville de la banlieue nord-ouest de Tokyo où l'on trouve le Mori Ghibli Bijutsukan (musée Ghibli).

34. Hayao Miyazaki, op. cit., p. 436.

\section{RÉSUMÉS}

Au Japon, rien n'est plus différent du reste du monde que l'aspect économique de la production des séries d'animation télévisées. Alors qu'en Europe, les sociétés de production attendent souvent des chaînes qu'elles s'engagent financièrement dans la réalisation des films, le Japon ne peut guère compter que sur un licensing particulier qui infléchit l'ensemble de la production et, ce faisant, le paysage télévisuel. Cette étude tente de déterminer par quels mécanismes cette situation s'est installée durablement dans l'archipel depuis les années 1960 et présente quelques dispositifs qui permettent à la télévision japonaise de lutter contre le nivellement mercatique des programmes par les multinationales du jeu et du divertissement.

In Japan, the economic aspects of the production of animated tv-series is highly different than in the rest of the world. While in Europe, production studios often expect that channels bring financial commitments to produce the film, Japanese artists can only depend on a particular licensing system that affects the whole production and, by this way, the television landscape. This study tries to determine the mechanisms by which this situation became permanent since the 1960s and presents some facilities that allow Japanese television to reject the marketing leveling of programs by gaming and entertainment companies. 


\section{AUTEUR}

\section{SAMUEL KACZOROWSKI}

Réalisateur de films d'animation, chercheur associé au LARA-SEPPIA (Université de Toulouse Jean-Jaurès) et agrégé d'arts appliqués. Il a consacré huit années à l'étude de l'œuvre filmique d'Osamu Tezuka à l'université Tokyo Hosei et en immersion dans différents studios d'animation (Ghibli, Mushi productions, Satelight, Ankama...). En 2017, il publie un ouvrage adapté de sa thèse : Capter le moment fuyant - Osamu Tezuka et l'invention de l'animation télévisée (Paris, L'Harmattan). Aujourd'hui, ses activités, partagées entre pratique et théorie, s'attachent aux problèmes de la profondeur dans le dessin animé, de la création d'un territoire commun entre animation, motion design et graphisme, et de l'écriture d'une histoire du cinéma d'animation via la technique. 